\title{
Association of age with left ventricular volumes, ejection fraction and concentricity: the Framingham heart study
}

\author{
Michael L Chuang ${ }^{1,2^{*}}$, Philimon Gona ${ }^{1}$, Gilion Hautvast ${ }^{3}$, Carol J Salton ${ }^{2}$, Marcel Breeuwer ${ }^{3}$, \\ Christopher J O'Donnell ${ }^{1}$, Warren J Manning ${ }^{2}$
}

From 16th Annual SCMR Scientific Sessions

San Francisco, CA, USA. 31 January - 3 February 2013

\section{Background}

Left ventricular (LV) parameters may vary with aging, but concomitant disease can obscure relationships between age and LV size, mass and function. CMR provides reproducible measures of LV volumes, mass, and ejection fraction (EF), but manual analyses are time consuming. Automated border detection (ABD) methods may decrease analytical burden. We sought to examine the effect of greater age on LV volumes, mass, EF and concentricity in a large cohort of healthy adults free of clinical cardiovascular disease and hypertension using ABDassisted methods.

\section{Methods}

1494 members of the Framingham Heart Study Offspring cohort, serially followed since 1971, underwent cine SSFP CMR (TR/TE/FA=3.2 ms/1.6 ms/60deg; $10-\mathrm{mm}$ thick contiguous short-axis slices) of the left ventricle at $1.5 \mathrm{~T}$. LV endo- and epicardial contours were automatically segmented by software (Extended MR Workspace 2009, Philips Healthcare), after which the operator had the option to adjust contours (if needed), followed by manual definition of the most basal slice at end-diastole. Concentricity was defined as LV mass/EDV (end-diastolic volume). We then identified a subset of healthy referent Offspring $(\mathrm{N}=685)$ free of any history of myocardial infarction, heart failure, CMR wall motion abnormality, and hypertension ( $\mathrm{SBP} \geq 140$ or $\mathrm{DBP} \geq 90 \mathrm{mmHg}$ or antihypertensive medication use) and stratified by decade age $(<50,50-59,60-69, \geq 70$ years). We compared sexes by 2 -sample $t$ test and used linear regression to test for within-sex trend across age groups. We assessed observer reproducibility $(\mathrm{n}=48)$ by intraclass correlation coefficient (ICC).

\section{Results}

Referent participants were aged $61 \pm 9$ years $(262$ men, 423 women). Men had greater LV volumes, mass and concentricity than women $(\mathrm{p}<0.0001$, all), but women had greater $\mathrm{EF}(73 \pm 6$ vs. $71 \pm 6 \%, \mathrm{p}=0.0002)$. As the Table (mean \pm SD) shows, LV EDV, end-systolic volume (ESV), and stroke volume (SV) decreased with greater age in both sexes; LV mass decreased with age in women but not men. After indexation to body surface area (BSA) trends in volumes remained, but mass/BSA did not trend with age in either sex. Both LV EF and concentricity increased with age in both sexes. ABD-assisted analysis was highly reproducible with interobserver $\mathrm{ICC}=0.99$, 0.98 for EDV and ESV, 0.99 for LV mass, and 0.96 for EF.

\section{Conclusions}

In a cohort of adults strictly free of clinical cardiovascular disease and hypertension, LV volumes, but not mass, decreased with greater age. LV concentricity and ejection fraction increased with age. Whether the increase in concentricity represents normal healthy aging or subclinical disease remains to be investigated.

\section{Funding}

Supported in part by NIH grant RO1 AG17509 and subcontract N01-HC-38038, and by an unrestricted educational grant from Philips Healthcare.

${ }^{1} \mathrm{NHLBI}$ 's Framingham Heart Study, Newton, MA, USA

Full list of author information is available at the end of the article

(c) 2013 Chuang et al; licensee BioMed Central Ltd. This is an Open Access article distributed under the terms of the Creative Commons Attribution License (http://creativecommons.org/licenses/by/2.0), which permits unrestricted use, distribution, and reproduction in any medium, provided the original work is properly cited. 
Table 1

\begin{tabular}{|c|c|c|c|c|c|}
\hline & $P$ (trend) & $<50 y$ & $50-59$ y & $60-69 y$ & $\geq 70 y$ \\
\hline MEN, N & & 26 & 104 & 94 & 38 \\
\hline $\mathrm{EDV}, \mathrm{ml}$ & $<0.0001, \downarrow$ & $168 \pm 28$ & $155 \pm 27$ & $145 \pm 29$ & $136 \pm 28$ \\
\hline $\mathrm{ESV}, \mathrm{ml}$ & $<0.0001, \downarrow$ & $53 \pm 16$ & $47 \pm 13$ & $41 \pm 14$ & $37 \pm 10$ \\
\hline $\mathrm{SV}, \mathrm{ml}$ & $0.005, \downarrow$ & $109 \pm 18$ & $109 \pm 19$ & $103 \pm 20$ & $99 \pm 21$ \\
\hline Mass, $g$ & 0.36 & $94 \pm 13$ & $103 \pm 20$ & $96 \pm 22$ & $97 \pm 20$ \\
\hline EDV/BSA, $\mathrm{ml} / \mathrm{m}^{2}$ & $0.0001, \downarrow$ & $79 \pm 12$ & $76 \pm 13$ & $71 \pm 14$ & $69 \pm 14$ \\
\hline $\mathrm{ESV} / \mathrm{BSA}, \mathrm{ml} / \mathrm{m}^{2}$ & $<0.0001, \downarrow$ & $26 \pm 7$ & $23 \pm 6$ & $21 \pm 7$ & $19 \pm 5$ \\
\hline $\mathrm{SV} / \mathrm{BSA}, \mathrm{ml} / \mathrm{m}^{2}$ & $0.03, \downarrow$ & $54 \pm 9$ & $53 \pm 9$ & $51 \pm 9$ & $50 \pm 11$ \\
\hline Mass/BSA, $\mathrm{g} / \mathrm{m}^{2}$ & 0.73 & $46 \pm 5$ & $50 \pm 9$ & $47 \pm 9$ & $49 \pm 9$ \\
\hline Mass/EDV & $0.0036, \uparrow$ & $0.77 \pm 0.12$ & $0.84 \pm 0.17$ & $0.85 \pm 0.16$ & $0.92 \pm 0.30$ \\
\hline$E F, \%$ & $<0.0001, \uparrow$ & $68 \pm 6$ & $70 \pm 6$ & $72 \pm 6$ & $73 \pm 5$ \\
\hline Women, N & & 24 & 179 & 139 & 81 \\
\hline $\mathrm{EDV}, \mathrm{ml}$ & $<0.0001, \downarrow$ & $127 \pm 22$ & $116 \pm 21$ & $108 \pm 19$ & $103 \pm 17$ \\
\hline ESV, ml & $<0.0001, \downarrow$ & $38 \pm 10$ & $33 \pm 9$ & $30 \pm 9$ & $25 \pm 7$ \\
\hline $\mathrm{SV}, \mathrm{ml}$ & $<0.0001, \downarrow$ & $89 \pm 17$ & $83 \pm 16$ & $78 \pm 13$ & $78 \pm 13$ \\
\hline Mass, $\mathrm{g}$ & $0.009, \downarrow$ & $68 \pm 18$ & $59 \pm 13$ & $56 \pm 11$ & $28 \pm 11$ \\
\hline EDV/BSA, $\mathrm{ml} / \mathrm{m}^{2}$ & $<0.0001, \downarrow$ & $69 \pm 8$ & $66 \pm 10$ & $62 \pm 9$ & $61 \pm 9$ \\
\hline ESV/BSA, $\mathrm{ml} / \mathrm{m}^{2}$ & $<0.0001, \downarrow$ & $21 \pm 5$ & $19 \pm 5$ & $17 \pm 5$ & $15 \pm 4$ \\
\hline $\mathrm{SV} / \mathrm{BSA}, \mathrm{ml} / \mathrm{m}^{2}$ & $0.02, \downarrow$ & $48 \pm 6$ & $47 \pm 8$ & $45 \pm 7$ & $46 \pm 7$ \\
\hline Mass/BSA, $\mathrm{g} / \mathrm{m}^{2}$ & 0.27 & $37 \pm 7$ & $34 \pm 6$ & $33 \pm 5$ & $34 \pm 6$ \\
\hline Mass/EDV & $<0.0001, \uparrow$ & $0.70 \pm 0.09$ & $0.72 \pm 0.86$ & $0.74 \pm 0.11$ & $0.78 \pm 0.12$ \\
\hline$E F, \%$ & $<0.0001, \uparrow$ & $70 \pm 6$ & $71 \pm 6$ & $73 \pm 5$ & $76 \pm 5$ \\
\hline
\end{tabular}

\section{Author details}

${ }^{1} \mathrm{NHLBI}$ 's Framingham Heart Study, Newton, MA, USA. ${ }^{2}$ Cardiovascular Division, Beth Israel Deaconess Medical Center, Boston, MA, USA. ${ }^{3}$ Imaging Systems, Philips Healthcare, Best, Netherlands.

Published: 30 January 2013

\section{Submit your next manuscript to BioMed Central} and take full advantage of:

- Convenient online submission

- Thorough peer review

- No space constraints or color figure charges

- Immediate publication on acceptance

- Inclusion in PubMed, CAS, Scopus and Google Scholar

- Research which is freely available for redistribution

Submit your manuscript at www.biomedcentral.com/submit 